\title{
Exploring the Experiences of Chinese Patients Waiting for Heart Transplantation
}

\author{
Hongxia Song1 ${ }^{1}$ Jianhong Qiao ${ }^{2}$, Yunfeng Li², Mei Han ${ }^{2 *}$ \\ ${ }^{1}$ Cardiology Department, Shandong Provincial Qianfoshan Hospital, The First Affiliated Hospital of Shandong First Medical \\ University, Ji'nan, China \\ ${ }^{2}$ Department of Nursing, Shandong Provincial Qianfoshan Hospital, The First Affiliated Hospital of Shandong First Medical \\ University, Ji'nan, China \\ Email: xysong1983@foxmail.com, ^493472456@qq.com
}

How to cite this paper: Song, H.X., Qiao, J.H., Li, Y.F. and Han, M. (2021) Exploring the Experiences of Chinese Patients Waiting for Heart Transplantation. World Journal of Cardiovascular Diseases, 11, 153-166. https://doi.org/10.4236/wjcd.2021.113017

Received: February 3, 2021

Accepted: March 19, 2021

Published: March 22, 2021

Copyright $\odot 2021$ by author(s) and Scientific Research Publishing Inc. This work is licensed under the Creative Commons Attribution International License (CC BY 4.0).

http://creativecommons.org/licenses/by/4.0/

\begin{abstract}
Background: Patients waiting for heart transplantation (HT) have complex physiological and psychosocial problems. Factors such as psychological state, behavior, social relationships, and cultural background of patients influence the HT process, such as causing psychotic disorder, difficult decision and close dependence. Care during the waiting period needs to be aimed at not only treating the symptoms but also the specific status. Purpose: To describe the physical and psychosocial experiences of the patients waiting for HT in one general hospital in the northern part of China. Methods: A qualitative approach was used in this study. Fifteen patients waiting for HT were selected based on purposive sampling, and semi-structured interviews were carried out. Results: In the analysis process, reported experiences of the participants were categorized into five main themes as follows: 1) feeling the impact; 2) uncertainty about the future; 3) difficult to make decisions; 4) negative emotions and 5) request support. Conclusion: This study provides an in-depth description of patients waiting for HT who have experienced many physical, psychosocial, and familial problems due to disease and their cultural background. It found that Chinese patients have some special experiences due to their psychosocial background. This study suggests that nurses should focus on not only physical but also psychosocial problems of these patients, and provide understandings to develop more effective strategies to solve their problems. Relevance to Clinical Practice: This study focused on the special patients who are waiting for heart transplantation, and got the special results about the feelings and experiences. The results can help the doctors and nurses to help the patients pass the special period smoothly.
\end{abstract}

\section{Keywords}

Heart Transplantation, Qualitative Research, Patient Experience, Thematic 
Analysis, Nursing

\section{Introduction}

End-stage heart disease has very high morbidity and mortality that increase health-care costs and leads to worsened quality of life, due to heart failure (HF) [1] [2], despite active medical and mechanical treatment. Approximately 26 million people worldwide and 6.5 million people in the United States are affected by HF [3] [4]. Hospitalization rates are high in the first decade of 21th century in the US and Europe, the prevalence of patients living with HF will increase by $46 \%$ by the year 2030, achieving epidemic proportions [5] Although survival of patients with HF has improved due to advances in therapy, the death rate remains at approximately $50 \%$ within 5 years from the time of diagnosis [4]. Consequently, long term survival remains poor in patients with end-stage heart disease [2].

Heart transplantation (HT) is considered to be the gold standard treatment and the only effective procedure in patients with end-stage heart disease [6]. Many patients who have end-stage heart disease recognize that HT as a "second chance" or "gift of life" [7]. However, most patients have to keep living with complex medical therapies and mechanical support due to the lack of donors. Worldwide, approximately $24 \%$ of transplant candidates die before a heart becomes available [8].

In 2014, China announced cease the use of organs from executed prisoners. This means all heart transplantations in China now rely on donation after brain death. According to a report by the Chinese Organ Transplantation Network in 2019 , the number of registered organs transplantation performed in the mainland of China had reached 300 thousand by June 2019, and 1200 of these cases were performed in 2019. The hospital where the researchers worked located in the north part of China which serves almost 100 million people, over $100 \mathrm{pa}$ tients per year wait for heart transplantation, however, only about 20 - 30 donated hearts are available for HT. The shortage of donor organs is the major limitation for all forms of organ transplantation. Consequently, patients must to wait for a donated heart for a much longer time than previously.

HF has caused many symptoms such as dizzying, fatigue and dyspnea [9], which have a strong impact on the patients' quality of life [7]. Importantly, dyspnea and fatigue may limit exercise tolerance and fluid retention. On the other hand, Anticipating challenges after transplant such as debilitating side effects, variable pharmacokinetics, rejection, malignancy and infection risk are valid concerns and may affect patients pre-transplant psychological status [10].

Previous studies reported that pre-transplant psychological status predicts posttransplant psychological status, length of hospitalization, and the success of surgery [11] Thus, understanding the psychological status in patients waiting for HT can help to predict and address the mental health and psychosocial needs of 
post-surgery rehabilitation. Quantitative research has focused on psychological distress, anxiety and stress disorders of the patients who are undergoing HT in many developed countries for many years [6] [8] [12]. One research has evaluated country level socioeconomic characteristics and explained geographic disparities and influence outcomes of patients undergoing HT. Community socioeconomic background may influence access to care and social support available to transplant recipients, whereas individual disadvantage due to socioeconomic status may increase patients' morbidity and psychological burden [13]. The country of China shows some different characteristics from the western countries, such as health care system, value orientation and the way of doing things by the religious influence including Confucianism and Buddhism [14]. Therefore, according to the different cultural and socio-economic backgrounds, we explored the experiences of Chinese patients when waiting for donated hearts in hospital.

The objectives of this study were:

1) To describe the feelings and experiences of the Chinese patients waiting for heart transplantation with a Chinese community socioeconomic background.

2) To discuss proposed intervention methods that nurses can provide to these patients according to their physiological and psychosocial needs.

\section{Methods}

\subsection{Approach}

This qualitative study accessed and analysed the viewpoint of patients with regard to their perception of illness and the therapeutic strategies when they used waiting for HT. Data was coded within a thematic analysis which label a condensed meaning unit to allow the data to be thought about in new and different ways [15]. Codes including verbal, non-verbal expression (mood, intonation, facial expression and gestures) were grouped thematically [16]. In this study, an initial thematic analysis led to successive level of depth and meaning.

\subsection{Participants}

This study was conducted between May 2018 and Aug 2019. Patients waiting for HT were invited to attend this study after the researcher explained the objective, procedure and so on.

The researchers interviewed patients one by one. Data was analyzed immediately after the interview finished. The participators were invited into the research continue until the results become saturated, repetition which means the sample the sample selected could represent the whole population. Participants in this study included 15 inpatients referred for HT at a general hospital in the north part of China in the end. Voluntary participations signed the informed consent before the interview begin. Basic demographic information (relating to the age, gender, education, marital status and insurance condition) is shown in Table 1.

Inclusion criteria were as follows: 1) has been diagnosed with end-stage heart disease by cardiovascular expert, 2) is waiting for a donor, 3) has ability to express 
their experiences, 4) is vital signs stable and no psychotic disorder as determined by attending doctors and psychologist, and 5) is willing to participate the study and signed informed consent. Exclusion criteria were as follows: 1) vital signs were not stable, 2) evidence of mental health issues were diagnosed by psychologist.

\subsection{Data Collection}

Interviews were held in a quiet room or the bed of patients at the HT unit. Three of the interviewers are nursing specialist and who have been nursing cardiac patients for 7 years at least. The main method of data collection in this research was in-depth semi-structured interviews, which take between 30 - 45 minutes. The interview outline was developed by the research team. Participants were asked to express their understanding of their experience when waiting for HT. Questions began with: "What are your experiences during this period?" and "What do you think about your disease?" with follow up questions as necessary. The interview continued to obtain a in-depth understanding of what they were experiencing. Fellow up questions included: "Can you tell me more about that?" "How do you deal with this situation?" Interviews were recorded by digital voice recorder. During the interviews, researcher also took field notes. Sampling continued until the results become rich, meaningful, accurate and saturated [17].

Table 1. The basic information of the participants.

\begin{tabular}{|c|c|c|c|c|c|c|c|}
\hline Number & Gender & Age $(Y)$ & Original disease & Marital status & NYHA & Level of education & Insurance conditions \\
\hline 1 & male & 28 & $\mathrm{DCM}$ & married & IV & High school & $\mathrm{NCM}$ \\
\hline 2 & male & 31 & DCM & married & III & High school & City medical insurance \\
\hline 3 & female & 32 & DCM & married & III & Middle school & $\mathrm{NCM}$ \\
\hline 4 & male & 52 & DCM & married & IV & Middle school & City medical insurance \\
\hline 5 & male & 47 & DCM & married & III & University & Provincial medical insurance \\
\hline 6 & male & 22 & DCM & single & IV & Middle school & $\mathrm{NCM}$ \\
\hline 7 & female & 61 & IHD & married & IV & University & Provincial medical insurance \\
\hline 8 & female & 33 & DCM & married & III & Middle school & NCM \\
\hline 9 & male & 34 & DCM & divorced & III & Middle school & NCM \\
\hline 10 & male & 61 & IHD & married & III & Middle school & NCM \\
\hline 11 & male & 56 & DCM & divorced & III & University & City medical insurance \\
\hline 12 & male & 69 & IHD & married & IV & Primary school & $\mathrm{NCM}$ \\
\hline 13 & male & 35 & $\mathrm{DCM}$ & married & IV & Middle school & $\mathrm{NCM}$ \\
\hline 14 & male & 42 & DCM & married & III & High school & Commercial health insurance \\
\hline 15 & female & 39 & AHMD & married & III & University & Provincial medical insurance \\
\hline
\end{tabular}

PS: Dilated cardiomyopathy (DCM); Ischemic heart disease (IHD); Alcoholic cardiomyopathy (AHMD); New rural cooperative medical (NCM); NYHA: New York Heart Association Class. 


\subsection{Data Analysis}

Phenomenological approach analysis was used to analyze the data. The procedures were divided into seven steps: reading the interview data and listening to the recording; transcribing and recording the nonverbal behavior; extracting units of analysis; abstracting and labeling with a code; formulating into themes and verifying by the member checking. Abbreviations were used, for example, Case1, session1 (C1S1 for short).

\subsection{Ethical Consideration}

The present research was approved by the Ethics Committee of Hospital. All participants signed the informed consent before the interview. The research team explained the research methods and objectives to the participants and promised that all the participants could withdraw at any stage of the study without any consequences.

\subsection{Data Trustworthiness}

Strategies were used to ensure rigor and trustworthiness of the data and analysis included 1) use of multiple sites for recruitment, 2) tactics was used to encourage the participants frank from the outset of each session, 3) Semi-structured questioning techniques, 4) collaborative sessions were used to discuss alternative approaches, 5) an iterative analysis process to track the decision making process, 6) review and discussion with three co-researcher, and 7) member check to verifying the authenticity of the themes.

\section{Results}

\subsection{Demographics and Overview}

The participants' age range was $22-69$ years old $(42.80 \pm 14.10)$. A total of 15 patients were interviewed, among whom 11 were men and 12 were married. The gender between male and female are are imbalance. The specifications of the participants are showed in the Table 1. Five main themes comprised "Feeling the impact," "Uncertainty about the future," "Difficult to make decisions," "Negative emotions," and "Request support" were emerged from data analysis, which were the result of experience of patients who are waiting for HT in China. The meanings of each of these themes have been provided using direct quotes of the participants below.

\subsection{Theme 1: Feeling the Impact}

End-stage heart disease has caused many symptoms and have a strong impact on the patients' quality of life. Most of the patients reported "feeling the impact", with its constituent themes of "Physical limits," and "Discomfort" of the clinical symptom and emotional effects of the heart illness, as well as aspects of dyspnea, fluid restrictions and discomfort, typically linked in some treatment methods to 
keep heart work and vital signs stable. It carried a sense of depression, with unpleasant or unwelcome doubt of self-worth. The sense crossed both physical and emotional levels.

"I feel so tired, I can't breathe, and I can't even leave my bed... all of this makes me very depressed, even anger. I often lose my temper. I' $s$ a torture if I keep going on living like this." (C7SI)

"I am limited on the amount of water which makes me uncomfortable. I feel anxiety... I mean, what is the meaning of those restrictions if I cannot live long enough to get a new heart. (C14S4)

This participant's reported the bad impact from clinical symptoms, which caused negative emotion. Beside the clinical symptoms can impact patients physiological, Some situation when they hospitalized led them to doubt their self-worth. Many participants feel discomfort referred to problems such as privacy, self-esteem due to their heart situation or treatment requires. Most of the participants who had expressed those problems were younger patients and women.

"I can't take care of myself, even the basic living skills. I feel painfully, because I can't just do anything that I can did before... You know, I even need nurses to help me when I am shitting. That leads me to feel shy and depressed." (C6S3)

"I was infused many kinds of medicines the whole day. Sometimes I cannot even wear clothes because of edema. A few days ago I had a catheter inserted, I felt almost naked at that time." (C8SI)

\subsection{Theme 2: Uncertainty about the Future}

This theme described patients' thought about the future. Worrying about the rejection reaction and the financial burden have affected the patients' mental health. They have no confidence for future life.

“...You know, my financial insurance is not very good, I have paid a lot of money for my disease. I have no confidence to earn enough money to pay for my debt when I discharge". (C13S1)

Returning to employment was important for some of participants especially for the young and that patients who used to have a good job, because illness had stopped them from working. Worry about work opportunity also influence their confidence for the future. Just like case 15 said:

"My Company has employed a new guy to do my work in my office... I think I can't go to work again." (C15S3)

Some patients described organ rejection after transplantation. Organ rejection is one of the most common complications of heart transplantation, which might result in the failure of the surgery. Considering the possibility of rejection reac- 
tion and the money that should pay for the anti-rejection drug affect the patients' confidence in the future. Experiences of one of the participants were as follows:

“...I know, I have to take anti-rejection drugs during the rest of my life after surgery, which also means a great amount of expense. Most important, I don't know if my body can accept my new heart successfully." (C6S4)

Such feeling of worry emerged of many of the participants in this research project included challenge (C14S2), terrible barrier (C1S4), the next 50 steps of the long march (C11S3).

\subsection{Theme 3: Difficult to Make Decisions}

Participants discussed how difficult to make a decision to prepare the transplantation. Some of them have decision-making difficulties because of the complexity of the problem.

"You know, made this decision is not an easy work. A few months ago, my doctor has talked to me that my heart condition will become worse and worse except change a new one. My families and I have considered all the possibility. To be honest, I was hesitating to give up or persist until that day I made the final decision." (C14S2)

"I decided to accept heart transplantation when I felt I was at death's door, but then hesitated when I felt better, time and time again." (C12S2)

Similarly, unresolved emotion emerged in the interview of many of the participants in this research. Besides the patients' complicated mentality, others relatives (parents, children, family members) are also to be considered.

"My parents made the decision for me... me? I agree, of course. You know, my life is not just mine. Cure me, stay with me is the spiritual forces of my parents." (C8S1)

"I want to give up when I feel more uncomfortable... I can't endure so much pain... but my little girl only twelve months old. To stay with her as long as possible is my expectation (sob)." (C1S3)

Even though the patients have made the decision to do the surgery, some of them appeared to be ambivalent.

"You know, my family and I have been waiting for a suitable heart for a long time. $I$ was not excited but scared when I was told 'to be prepared for the surgery'... I am a weirdo, isn't 1 ? When the doctor told me the procedure was aborted because the heart was not suitable, I was even relieved." (C2S3)

\subsection{Theme 4: Negative Emotions}

In this study, patients reported the negative emotions they were experiences 
while when they were waiting for heart transplantation, with its constituent sub themes of "passive", "guilt", "blame" and "dependence". This emergent theme involved patients responses to private and social relatives. It involved the view of themselves and their body and how it felt to face it.

Some patients acted as passive information consumers and rarely express an opinion about the doctor's suggestions. The experiences of some of the participants were as follows:

"Now, I stop thinking anything, the only thing I do now is chant sutras every day... it is my destiny." (C7S4)

"My doctor wants to talk to me, he said he needs to give me some information about the surgery and other treatment methods, looks like 'pacemaker' or something. Actually, I am not interested in the details."(C4SI)

The disease progresses had affected the family's members' daily life and increase burden to all aspects of their life (physical, mental, spiritual and economic). Therefore, many participants have shown the guilt of their family. Experiences of some of the participants were as follows:

“...I am over30 years old, I can't earn money, I have no ability to take care my old parents and bring up my little girl... ( silence)." (C2S2)

"My little daughter gives me water and tries to take care of me, but she just a little child needs her dad to take good care of her." (C1S1)

"Disease has destroyed me. I became angry, anxious and aggressive toward the people around me especially my family. I feel very sorry when I calm down." (C10S3)

Some participants believed that their heart disease was hereditary. They were afraid and concerned about the future of their children.

"I worry about my little child. I can't stop thinking of the chance that he will inherit my disease." (C13S4)

Likewise, Participant 3 outlined his guilty experience about the chance of inherit.

"Actually, I don't care how I might to be. I only want to pray for my children and hope their genes are clean. (C3S2)

One of the reasons of end-stage heart disease is hereditary or the progress of some "trivial disease". Some patients vent their anger on their parents because of this. Among them, the patients who had suffered the disease at early age got angry at their families. Experiences of one of the participants were as follows:

“... I blame them (his parents), or you can say I hate them. I got dilated cardiomyopathy because of the complications after viral cold when I was young. They didn't do their duty as parents (smiled awkwardly)." (C3S3)

Seeking more intimate relationships, especially the excessive reliance on fami- 
ly relationships were among the experience of participants. Some of the participants expressed dependence to family members.

"I asked my daughter to search information about the disease and food might be beneficial to me from internet. She cooks food in her home and brings it to the hospital for me." (C11S2)

"Nurses don't let my family and friends stay with me for a long time. I protested because I want my husband and my daughter to stay with me always... I am afraid I won't have a chance to say goodbye to them (sobs). (C8S3)

\subsection{Theme 5: Request Support}

This theme described the relationship that patients wanted to build between themselves and public organizations. The grand theme "request support," with its constituent themes of "financial support" and "social support".

Participants experienced different financial problems related to cost of the disease. Most of the participants who had experienced such problems were the patients who have no insurance or just basic Chinese Insurance-New rural cooperative medical (NCM). The experiences of two of the participants were:

"I have been in the hospital for six times since May last year until now. To be honest, I have a huge financial burden... You know, the payment from my insurance company is only $30 \%$ every time."(C1S4)

“...We got help from a charitable aid foundation when my mom did her eye operation. My dad has asked for help from the Chinese Red Cross. Unfortunately, it s only has a foundation for children who have congenital heart disease." (C3S2)

In China, most of the patients who are on the waiting list chose to stay in the hospital to wait for their donor. Actually, the heart transplant centers are located in some bigger cities, living expenses in these cities increase financial burden of the patients. The experience of one of the participants was:

"My dad bought a cooked fish from a restaurant in order to supply protein for me. You cannot believe that he must pay 17 dollars for the fish. The living expenses are too high to afford it for us." (C9S3)

Some patients were asking for social support and sought some support to solve their families' difficulties.

"My mom has eye disease, my wife has to take care our little girl at home. I hope government departments can give us some help, such as offer a suitable part-time job for my wife and my mom." (C3S2)

Many patients prefer to relate to the members who experienced the same illness and some of the patients expressed their needs from the policy.

"I would like to communicate with the people who have the same disease or 
have undergone the surgery. I often chat in our Wechat group, in which all the members are patients waiting for heart transplantation or have finished the surgery, it is more like our home." (C11S2)

"I can't get the medical aid, according to the serious illness hospitalization insurance, just because the heart transplantation is not in the system catalog. The government departments should reconsider it." (C12S4)

\section{Discussion}

A total of 15 patients participanted our study, included 11 males and 4 females. Experiences of the participants were divided into five main categories: feeling the impact, uncertainty about the future, difficult to make decisions, negative emotions and request supports.

The period of waiting for HT is extremely stressful, and might involve physical pain, economic and spiritual pressure, feeling "worthless" and even fear of the bad situation of employment and so on [18]. This intense period are common before transplantation [19], and these symptoms, along with lower QOL, are affects patients' psychosocial well-being. Research reported that pre-transplantation cardiac patients both still waiting at home and hospitalized have experienced negative emotions similar to our study [12]. Rodrigue [20] described these patients' limitations in daily life and reduced quality of life owing to the physiological symptoms. However, patients have different characteristics due to the different socialeconomic background, for example, unlike other research, Ye [21] found that anxiety symptoms are more prevalent and more severe than other psychological symptoms among inpatients waiting for heart transplantation in China.

Rejection rates are higher among younger adults, whereas morbidities in order adults include infection, renal failure and malignancy [22]. Both of the risk factors and the lifestyle changes are make patients of all ages uncertainty regarding future life and health. In our study, we think financial burden to be one of the characterizes of Chinese patients play an important role that led patients uncertainty for the future, because most of them just have a basic health insurance which can't good cover the fees of surgery and rejection medicines. Meanwhile, Only few qualified hospitals can do heart transplant in China. The hospital in this study receives patients from all over the country. The present reimbursement regulation is that the patients get the medical treatment and pay the full cost, and then submit the expense account to the medical organization for the patients from other cities especially for the patients with new rural cooperative medical insurance.

In this study, the patients expressed the special experiences of psychological conflict and complex psychological responses to the family members, which we think is the result of the influence of Chinese' traditional value systems. In fact, it is recognized that disease management differs among ethnic and social classes [23]. What can we do in a different cultural background? Hence, listening to the patient's complaints, giving treatment to cure the patients' pain in time, and 
providing knowledge to the patients and the family members are useful methods for the patients to solve physical and psychological problems. In fact, medical staff spend very little time on the patients' psychological state in most Chinese hospitals. Hence, this is an important characteristic which should be drawn to the attention of Chinese medical workers. It has been shown previously that the active involvement of family members in patients' care can achieve good outcomes [24]. Sometimes, the family members act as a guide in the course of the patients' treatment, especially for Chinese patients who have specific family values. We suggest that patients' family members and caregivers come together and take more time to discuss the patients' situation and treatment strategy. Nurses can provide the patients and their family member some knowledge about the disease and heart transplantation and encourage them to attend the patients' management actively.

In our study, patients expressed the need of support, consistent with findings by Ivarsson [25], who reported that patients expressed positive views about the support and information they wanted to get from the society and healthcare professionals group. One of the characteristics of this study was that patients seek both financial support and social support. The participants with financial problems must focus on the patients whose insurance is not sufficient. They also faced many problems in their life that need help from some social agency. Social support can relieve stress effects and protect patients from stress-related factors [26]. Therefore, it is one of the signs that we should provide the patients with appropriate information according to their background. Richards [27] has indicated that social support from health care providers was positively for the patients to know their physical limitations and their progress in recovery, such as, set up a "network" or "big family" including nurses and other patients. One study [12] showed that nurses being easily available and keeping regular contact is of great importance to patients during a period of uncertainty and worry. Napolitano [28] has reported that a brief telephone-based psychological intervention can significantly improve wait-list transplantation patients' QOL and social support. Similarly, some researches have shown that social support from family and peers can help patients in physical activity [29].

\section{Conclusion}

Chinese patients waiting for HT have some special experiences including physical, psychosocial, and family problems due to disease and their psychosocial background which may seriously affects prognosis of patients. This research alerts the staff in the hospital need to learn the social contexts and pay more attention to the psychosocial problems of the patients waiting for HT. This research indicates that making effective interventions and establish social support system could help the patients.

\section{Limitation}

The participants' gender is imbalance in this study. Firstly, this study is very 
small, with a small number of patients, with rather short-term study. Secondly, the prevalent of HF are similar between the genders, but the progression of disease is slowly in female. The symptoms and complications of HF influences mortality in patients of both genders, but, this effect is weaker in women because of a difference in the oxygen affinity of hemoglobin. It is well documented that women patients of HF are more likely than men to be older and to have a preserved ejection fraction. Thirdly, we think is the influence of Chinese cultural. On one hand, many families especially in the rural area more like boy under the background of one child policy implemented in 1979. On the other hand, most of the families have new rural cooperative medical which is a low level coordination system. Hence, they prefer to spend money on boys.

\section{Relevance to Clinical Practice}

The findings of this study provide significant insight into the experiences of Chinese patients waiting for heart transplantation. Based on these findings, Chinese patients waiting for heart transplantation suffer many problems in different aspects of health such as physical, psychological, social and so on. These patients cope with their transplant process based on their cultural and social context. This finding implies the direction of intervention to the patients in the transplant process to solve the mental problems (e.g., insurance, information, and socioeconomic factors). It is recommended that further research should be done on patients waiting for heart transplantation in other cultures in order to tailor intervention methods with regard to cultural differences.

\section{Conflicts of Interest}

No conflict of interest exits in the submission of this manuscript, and manuscript is approved by all authors for publication.

\section{References}

[1] Theochari, C.A., Michalopoulos, G., Oikonomou, E.K., Giannopoulos, S., Doulamis, I.P., Aillela, M. and Kokkinidis, D.G. (2018) Heart Transplantation versus Left Ventricular Assist Devices as Destination Therapy or Bridge to Transplantation for 1-Year Mortality: A Systematic Review and Meta-Analysis. Annals of Cardiothoracic Surgery, 7, 3-11. https://doi.org/10.21037/acs.2017.09.18

[2] Aguanno, J. and Samson, L. (2018) Diagnosis and Management of Patients with Heart Failure. Medical Laboratory Observer, 50, 6-12. https://doi.org/10.1007/s10741-016-9587-y

[3] Myserlis, P.G., Malli, A., Kalaitzoglou, D.K., Kalaitzidis, G., Miligkos, M., Kokkinidis, D.G. and Kalogeropoulos, A.P. (2017) Atrial Fibrillation and Cognitive Function in Patients with Heart Failure: A Systematic Review and Meta-Analysis. Heart Failure Reviews, 22, 1-11. https://doi.org/10.1007/s10741-016-9587-y

[4] Benjamin, E.J., Blaha, M.J., Chiuve, S.E., Cushman, M., Das, S.R., Deo, R., et al. (2017) Heart Disease and Stroke Statistics-2017 Update: A Report from the American Heart Association. Circulation, 135, e146-e603. https://doi.org/10.1161/CIR.0000000000000485 
[5] Heidenreich, P.A., Albert, N.M., Allen, L.A., Bluemke, D.A., Butler, J., Fonarow, G.C., et al. (2013) Forecasting the Impact of Heart Failure in the United States: A Policy Statement from the American Heart Association. Circulation: Heart Failure, 6, 606-619. https://doi.org/10.1161/HHF.0b013e318291329a

[6] Milaniak, I., Wilczek-Rozyczka, E., Wierzbicki, K., Piatek, J., Kedziora, A. and Przybylowski, P. (2018) The Effect of Clinical Variables on Distress and Depressive Symptoms among Heart Transplant Recipients. Heart and Lung, 47, 68-72.

https://doi.org/10.1016/j.hrtlng.2017.09.008

[7] Patricia, W. (2014) Health-Related Quality of Life and Mortality in Incident Heart Failure from the Cardiovascular Health Study (CHS). University of Maryland, Baltimore, 251.

[8] Conway, A., Sheridan, J., Maddicks-Law, J. and Fulbrook, P. (2016) Pilot Testing a Model of Psychological Care for Heart Transplant Recipients. BMC Nursing, 15, Article No. 62. https://doi.org/10.1186/s12912-016-0183-1

[9] Zambroski, C.H., Moser, D.K., Roser, L.P., Heo S. and Chung, M.L. (2005) Patients with Heart Failure Who Die in Hospice. American Heart Journal, 149, 558-564. https://doi.org/10.1016/j.ahj.2004.06.019

[10] Koomalsingh, K. and Kobashigawa, J.A. (2018) The Future of Cardiac Transplantation. Annals of Cardiothoracic Surgery, 7, 135-142. https://doi.org/10.21037/acs.2017.12.02

[11] Bunzel, B. and Wollenek, G. (1994) Heart Transplantation: Are There Psychosocial Predictors for Clinical Success of Surgery? Journal of Thoracic and Cardiovascular Surgery, 42, 103-107. https://doi.org/10.1055/s-2007-1016466

[12] Polastri, M., Savini, C., Di Marco, L., Jafrancesco, G., Semprini, A. and Grigioni, F. (2017) Post-Operative Pleural Effusion in a Heart Transplant Recipient: A SingleCase Study of Physiotherapy Treatment. International Journal of Therapy and Rehabilitation, 24, 302-305. https://doi.org/10.12968/ijtr.2017.24.7.302

[13] Tumin, D., McConnell, P.I., Galantowicz, M., Tobias, J.D. and Hayes Jr., D. (2017) Reported Non Adherence to Immunosuppressive Medication in Young Adults after Heart Transplantation: A Retrospective Analysis of a National Registry. Transplantation, 101, 421-429. https://doi.org/10.1097/TP.0000000000001152

[14] Williams, M.M., Foo, K.H. and Haarhoff, B.A. (2006) Cultural Considerations in Using Cognitive Behaviour Therapy with Chinese People: A Case Study of an Elderly Chinese Woman with Generalised Anxiety Disorder. New Zealand Journal of Psychology, 35, 153-162.

[15] Coffey, A. and Atkinson, P. (1996) Making Sense of Qualitative Data. Complementary Research Strategies. Sage Publications Inc., Thousand Oaks, London, New Delhi.

[16] Braun, V. and Clarke, V. (2006) Using Thematic Analysis in Psychology. Qualitative Research in Psychology, 3, 77-101. https://doi.org/10.1191/1478088706qp063oa

[17] Waldron, R., Malpus, Z., Shearing, V., Sanchez, M. and Murray, C.D. (2017) Illness, Normality and Identity: The Experience of Heart Transplant as a Young Adult. Disability and Rehabilitation, 39, 1976-1982. https://doi.org/10.1080/09638288.2016.1213896

[18] Castle, H. and Jones, I. (2004) A Long Wait: How Nurses Can Help Patients through the Transplant Pathway. Professional Nurse, 19, 37-39.

[19] Parekh, P., Blumenthal, J.A., Babyak, M., Merrill, K., Caeney, R.M., Davis, R.D. and Palmer. S.M. (2003) Psychiatric Disorder and Quality of Life in Patients Awaiting Lung Transplantation. Chest, 124, 1682-1688.

https://doi.org/10.1378/chest.124.5.1682 
[20] Rodrigue, J.R., Baz, M.A., Widows, M.R. and Ehlers, S.L. (2005) A Randomized Evaluation of Quality-of-Life Therapy with Patients Awaiting Lung Transplantation. American Journal of Transplantation, 5, 2425-2432.

[21] Ye, C.Y., Zhuang, Y., Zhang, Y., Lin, Y., Ji, J.L. and Chen, H. (2013) Anxiety, Depression, and Associated Factors among Inpatients Waiting for Heart Transplantation. Shanghai Archives of Psychiatry, 25, 165-173.

[22] Lund, L.H., Edwards, L.B., Kucheryavaya, A.Y., Dipchand, A.I., Benden, C. and Stehlik, J. (2013) The Registry of the International Society for Heart and Lung Transplantation: Thirtieth Official Adult Heart Transplant Report-2013; Focus Theme: Age. Journal of Heart and Lung Transplantation, 32, 951-964.

[23] Li, T.G. and Wang, M. (2017) Paying Attention to Different Health Needs of Different Ethnic Groups in Process Health for All Program. Chinese Journal of Epidemiology, 10, 693-695. [Original work published in Chinese]

[24] Mase, R., Halasyamani, L., Choi, H. and Heisler, M. (2015) Who Signs Up for and Engages in a Peer Support Heart Failure Self-Management Intervention. Journal of Cardiovascular Nursing, 30, S35-S43. https://doi.org/10.1097/JCN.0000000000000172

[25] Ivarsson, B., Ekmehag, B. and Sjöberg, T. (2011) Recently Accepted for the Waiting list for Heart or Lung Transplantation-Patients' Experiences of Information and Support. Clinical Transplantation, 25, E664-E671. https://doi.org/10.1111/j.1399-0012.2011.01511.x

[26] Won, M.H. and Son, Y.J. (2017) Perceived Social Support and Physical Activity among Patients with Coronary Artery Disease. Western Journal of Nursing Research, 39, 1606-1623. https://doi.org/10.1177/0193945916678374

[27] Richards, E.A. and Cai, Y. (2016) Integrative Review of Nurse-Delivered Physical Activity Interventions in Primary Care. Western Journal of Nursing Research, 38, 484-507. https://doi.org/10.1177/0193945915581861

[28] Napolitano, M.A., Babyak, M.A., Palmer, S. Tapson, V., Davis, D. and Blumenthal, J. (2002) Effects of a Telephone Based Psychosocial Intervention for Patients Awaiting Lung Transplantation. Chest, 122, 1176-1184. https://doi.org/10.1378/chest.122.4.1176

[29] Aliabad, H.O., Vafaeinasab, M., Morowatisharifabad, M.A, Afshani, S.A., Firoozabadi, M.G. and Forouzannia, S.K. (2014) Maintenance of Physical Activity and Exercise Capacity after Rehabilitation in Coronary Heart Disease: A Randomized Controlled Trial. Global Journal of Health Science, 6, 198-208.

https://doi.org/10.5539/gjhs.v6n6p198 